\section{LI-RADS ancillary features on contrast-enhanced ultrasonography}

\author{
Christoph F. Dietrich ${ }^{1,2}$, Yi Dong ${ }^{3}$, Yuko Kono ${ }^{4}$, Cosmin Caraiani ${ }^{5}$, Claude B. Sirlin 6 , \\ Xin-Wu Cui ${ }^{7}$, An Tang ${ }^{8}$ \\ 'Department Allgemeine Innere Medizin (DAIM), Kliniken Beau Site, Salem und Permanence, \\ Hirslanden, Bern, Switzerland; ${ }^{2}$ Ultrasound Center, The First Affiliated Hospital of Zhengzhou \\ University, Zhengzhou; ${ }^{3}$ Department of Ultrasound, Zhongshan Hospital, Fudan University, \\ Shanghai, China; ${ }^{4}$ Departments of Medicine and Radiology, University of California, San \\ Diego, CA, USA; ${ }^{5}$ Department of Medical Imaging, luliu Hatieganu University of Medicine \\ and Pharmacy, Cluj-Napoca, Romania; ${ }^{6}$ Department of Radiology, University of California \\ San Diego, San Diego, CA, USA; ${ }^{7}$ Department of Medical Ultrasound, Tongji Hospital, \\ Tongji Medical College, Huazhong University of Science and Technology, Wuhan, China; \\ ${ }^{8}$ Department of Radiology, Université de Montréal, Montreal, Canada
}

The Liver Imaging Reporting and Data System (LI-RADS) was created to standardize liver imaging in patients at high risk for hepatocellular carcinoma (HCC), and it uses a diagnostic algorithm to assign categories that reflect the relative probability of HCC, non-HCC malignancies, or benign focal liver lesions. In addition to major imaging features, ancillary features (AFs) are used by radiologists to refine the categorization of liver nodules. In the present document, we discuss and explain the application of AFs currently defined within the LI-RADS guidelines. We also explore possible additional AFs visible on contrast-enhanced ultrasonography (CEUS). Finally, we summarize the management of CEUS LI-RADS features, including the role of current and potential future AFs.

Keywords: Ultrasonography; Contrast-enhanced ultrasound; Hepatocellular carcinoma

\section{Introduction}

Hepatocellular carcinoma (HCC) is the second most lethal cancer, with a 6 -year survival rate of $18 \%$. It is the sixth most common cancer and the fourth most common cause of cancer-related death in the world [1]. The majority of HCCs are diagnosed via imaging without pathologic confirmation; thus, the accurate interpretation and reporting of imaging features are critical. The Liver Imaging Reporting and Data System (LI-RADS) was created to standardize the terminology, technique, interpretation, and reporting for HCC diagnosis in patients at risk for HCC. Earlier versions of the LI-RADS were developed for computed tomography (CT) and magnetic resonance imaging (MRI) (https://www. acr.org/Clinical-Resources/Reporting-and-Data-Systems/LI-RADS). Recently, LI-RADS algorithms and diagnostic tables have been developed for ultrasonography (US) and contrast-enhanced US (CEUS)

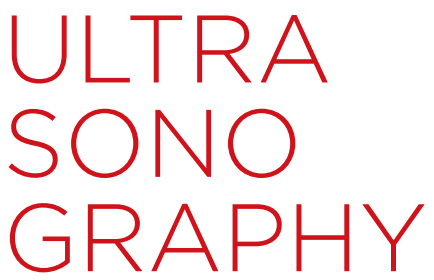

\section{REVIEW ARTICLE}

https://doi.org/10.14366/usg. 19052 pISSN: 2288-5919 elSSN: 2288-5943 Ultrasonography 2020;39:221-228

Received: October 1, 2019

Revised: April 2, 2020

Accepted: May 9, 2020

Correspondence to: Christoph F. Dietrich, MD, PhD, MBA, Department Allgemeine Innere Medizin (DAIM), Kliniken Beau Site, Salem und Permanence, Hirslanden, 3036 Bern, Switzerland

Tel. +41-76-440-8150

Fax. +41-31-337-6000

E-mail: c.f.dietrich@googlemail.com

This is an Open Access article distributed under the terms of the Creative Commons Attribution NonCommercial License (http://creativecommons.org/ licenses/by-nc/4.0/) which permits unrestricted noncommercial use, distribution, and reproduction in any medium, provided the original work is properly cited.

Copyright (C) 2020 Korean Society of Ultrasound in Medicine (KSUM)

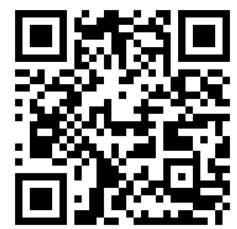

How to cite this article:

Dietrich CF, Dong Y, Kono Y, Caraiani C, Sirlin $C B$, Cui XW, et al. LI-RADS ancillary features on contrast-enhanced ultrasonography. Ultrasonography. 2020 Jul;39(3):221-228. 
[2-12]. According to the LI-RADS guidelines, major features are used to categorize observations as LR-3, LR-4, or LR-5.

The LI-RADS categorization scheme utilizes ancillary features (AFs) to improve detection, increase confidence, or adjust the category of the observation. These AFs may be applied at the interpreter's discretion $[13,14]$. The LI-RADS v2017 CEUS Core document, released by the American College of Radiology, briefly lists the application rules for CEUS AFs (https://www.acr.org/-/media/ACR/ Files/RADS/LI-RADS/CEUS-LI-RADS-2017-Core.pdf?la=en). Unlike in contrast-enhanced computed tomography (CE-CT) and contrast enhanced-magnetic resonance imaging (CE-MRI), in CEUS, AFs have generally not been used or accepted.

In this article, we review and illustrate AFs on CEUS, including their terminology, characterization, and possible applications.

\section{Definition of an Observation}

The LI-RADS approach defines an "observation" as an area that is distinct from the background liver parenchyma on imaging [15]. This generic term used for all imaging modalities is preferred over "lesion" or "nodule," since some observations, such as perfusion alterations and artifacts, may represent pseudolesions rather than true lesions or nodules. Hence, the term "observation" is used consistently in LI-RADS decision trees, algorithms, and tables. In clinical practice, narrower diagnostic terms may be alternatively used depending on the level of certainty. For example, the specific terms "simple cyst"; "solid hypoechoic," "hyperechoic," or "mixedechogenicity nodule"; or "thrombus in vein" may be used.

Observations can be either lesions or pseudolesions. While both are visible on imaging, lesions have a corresponding pathologic abnormality, whereas pseudolesions do not. Lesions include nodules, masses, confluent fibrosis or scarring, non-mass lesions (such as fat deposition or fatty sparing, iron deposition or sparing, and hemorrhage or edema), and treated lesions. Pseudolesions include artifacts, arterioportal shunts, and hypertrophic pseudomasses.

Some observations, such as nodules or masses, may be visible on all imaging modalities. Other observations are observed on a specific imaging modality or modalities, such as iron deposition on MRI. While the concept of observations has not typically been used in the context of CEUS, some phenomena described on CE-CT and CE-MRI can also be seen on CEUS, including pseudomasses, capsule vessels, and arterioportal shunts [16-18]. This is especially relevant in identifying the supplying vessels in focal fatty sparing or fat deposition at the periphery of the liver adjacent to the liver capsule.
Table 1. CEUS AFs recognized by the Liver Imaging Reporting and Data System

\begin{tabular}{ll}
\hline \multicolumn{1}{c}{ CEUS AFs favoring malignancy } & CEUS AFs favoring benignity \\
\hline Favoring malignancy in general, not & Size stability $\geq 2 \mathrm{yr}$ \\
HCC in particular & Size reduction \\
Definite growth & - \\
Favoring HCC in particular & - \\
Nodule-in-nodule appearance & - \\
Mosaic appearance & - \\
CEUS, contrast-enhanced ultrasound; AF, ancillary feature; HCC, hepatocellular \\
carcinoma.
\end{tabular}

\section{Definition of AFs}

AFs are defined by the LI-RADS guidelines as imaging features that modify the likelihood that an observation is HCC (Table 1). In isolation, these features do not permit the reliable categorization of observations and hence are considered ancillary. In prior versions of the LI-RADS, AFs were broadly divided into those favoring malignancy and those favoring benignity, and no rules for AF application were provided. In the 2017 version of the LI-RADS, AFs were divided into those favoring malignancy in general, those favoring HCC in particular, and those favoring benignity. The 2018 version of the LI-RADS guidelines then provided new rules regarding the application of AFs [13]. AFs should only be used if their presence is unequivocal; they should not be utilized if their presence is uncertain [19].

\section{AFs Favoring Malignancy in General}

Three CEUS AFs favor malignancy, one of which favors malignancy in general (definite growth) and two of which favor HCC in particular (nodule-in-nodule appearance and mosaic appearance). These AFs may be applied to upgrade the observation by one category to a maximum classification of LR-4. They cannot be used to upgrade the category to LR-5, and their absence should not be used to downgrade the LR category.

\section{Unequivocal Diameter Increase (Growth)}

Unequivocal diameter increase refers to an increase in the maximum diameter of a nodule over time which is not attributable to an artifact, differences in technique or modality between the exams, or measurement error $[13,20]$. Importantly, only US findings can be compared to US findings; while MRI and CT findings can each be compared to the other modality, neither should be compared to US results. Diameter change on US should be measured in B-mode. If the observation margins are unclear in B-mode, one may use CEUS 
images. If CEUS images are utilized, the interpreter should select enhanced images from the prior and current exams that show the lesion clearly and in the same phase; the arterial phase should be avoided, as it may lead to overestimation. Surrounding anatomical landmarks (the portal and hepatic veins, hepatic artery, gallbladder, and falciform ligament) should be used to ensure that the imaging planes used for observation measurements are comparable.

Diameter increase is an AF that favors malignancy but is not specific for HCC, since any malignant neoplasm is expected to grow [13]. The LI-RADS guidelines for CEUS do not stipulate a minimum increase in diameter required for use as an AF. If diameter increase is observed, interpreters may at their discretion upgrade the category by one level to a maximum classification of LR-4. Interpreters should exercise judgment in the application of this feature, which applies only in the case of an unequivocal increase in the diameter of an observation. Since threshold growth is not a major feature according to the LI-RADS CEUS guidelines, any unequivocal size increase is categorized as an AF favoring malignancy [13].

\section{AFs That Favor HCC in Particular}

The AFs that favor HCC in particular are mosaic appearance and nodule-in-nodule architecture (a subset of mosaic appearance). These features are characteristic of HCC but are rarely, if ever, observed in cholangiocarcinomas and other non-HCC malignancies; hence, they favor HCC in particular. These morphological patterns are best seen in the arterial phase, but the sizes of the internal nodules may vary depending on the exact timing of this phase.

\section{Mosaic Appearance}

Mosaic appearance is defined as the presence of randomlydistributed internal nodules or compartments, usually with different imaging features in terms of enhancement, attenuation, intensity, and size $[13,21]$. Mosaic appearance is thought to result from the clonal expansion of cells in various stages of dedifferentiation in the hepatocarcinogenesis pathway; for instance, one compartment may represent a precursor dysplastic nodule, while the other may contain early or progressed HCC. Imaging appearance is a reflection of the histology, with mosaic tumors comprising nodules with varying degrees of dedifferentiation, necrosis, fibrosis, cystic degeneration, and hemorrhage. The nodules are separated by thin septa or necrotic areas $[22,23]$. On imaging, mosaic appearance may be broadly categorized as follows:

- Nodule-in-nodule appearance, characterized by the presence of a smaller inner nodule (the daughter nodule) within a larger outer nodule (the parent nodule).

- Multi-nodule-in-nodule appearance, characterized by the presence of multiple inner daughter nodules within a larger parent mass.

- Multicompartment-in-nodule appearance, characterized by the presence of multiple discrete nodules and compartments randomly distributed within a larger mass.

- Septated solid mass, characterized by the presence of irregular enhancing internal septa [19].

Mosaic appearance is an AF that favors HCC. A mosaic mass that does not meet the LI-RADS major feature criteria for LR-5 (e.g., that lacks arterial phase hyperenhancement) cannot be categorized as LR-5. In patients with cirrhosis or other risk factors for HCC, however, most masses with a mosaic appearance can be categorized as LR-5 based on LI-RADS major features [19].

Mosaic appearance is a feature most commonly seen in tumors larger than $3 \mathrm{~cm}$. When assessing the size of an observation with a mosaic appearance, the entire mass should be measured, as opposed to measuring solely the internal nodules or the compartments with arterial phase hyperenhancement.

\section{Nodule-in-Nodule Appearance}

Nodule-in-nodule appearance refers to the presence of one or more inner nodules with imaging features that differ from those of a larger outer nodule [24]. Nodule-in-nodule appearance is a subset of mosaic appearance and is thought to result from the clonal expansion of cells that are at a more advanced stage of the hepatocarcinogenesis pathway than the cells of the outer nodule (Table 2). The inner nodule often exhibits features of a progressed HCC, whereas the outer nodule usually shows imaging features representative of a dysplastic nodule or an early HCC [25]. Nodulein-nodule appearance is a subtype of mosaic appearance; hence, the aforementioned description of that broader category largely applies. Regarding diameter measurement, for nodules with a nodule-innodule appearance, the entire observation should be included in the measurement, not just the internal nodule(s). An observation of nodule-in-nodule appearance that does not meet the major feature criteria for LR-5 cannot be categorized as LR-5. For such masses, a nodule-in-nodule appearance is an AF favoring malignancy. CEUS

Table 2. Summary of nodule-in-nodule appearance

\section{Appearance}

Nodule-in-nodule appearance specifically favors hepatocellular carcinoma as opposed to malignancy in general

Nodule-in-nodule appearance is a subset of mosaic appearance

Interpreters may, at their discretion, apply nodule-in-nodule appearance to upgrade the category (up to LR-4) for such observations

If uncertain about the feature, do not characterize it as having nodule-innodule appearance 
may show a nodule-in-nodule appearance more frequently than other modalities. While the internal and outer nodule are usually most obvious in the arterial phase, their sizes may vary depending on the exact timing of this phase. Therefore, size is measured more consistently on B-mode US and in the late phase than in the arterial phase.

\section{AFs Favoring Benignity}

AFs that favor benignity can be applied to downgrade the LI-RADS classification by one category. Their absence should not be used to upgrade the classification.

AFs favoring benignity are as follows: (1) unequivocal diameter reduction and (2) diameter stability for $\geq 2$ years.

\section{Diameter Stability for $\geq 2$ Years (Stable Size)}

Diameter stability is defined as:

- The lack of measurable change in the diameter of a nodule, measured on exams performed over 2 years or more and in the absence of treatment, or

- A change in diameter so small that the change is attributable to an artifact, differences in imaging technique or modality, or measurement error.

Diameter stability over 2 years or more in the absence of treatment is an AF that favors benignity. If this feature is observed, interpreters may at their discretion downgrade the LI-RADS classification by one category. After treatment, diameter stability should not be considered to be an AF favoring benignity.

\section{Unequivocal Diameter Reduction (Decreased Size)}

Unequivocal diameter reduction refers to a decrease in the maximum visualized diameter of a nodule measured over time that is not attributable to an artifact, differences in technique or modality between the exams, or measurement error. Decreased size applies to nodules that unequivocally become smaller in the absence of treatment (i.e., spontaneously). Diameter reduction in the absence of treatment is an AF that favors benignity. The LI-RADS guidelines for CEUS do not stipulate a minimum reduction in diameter required for use as an AF. If this feature is observed, interpreters may at their discretion downgrade the LI-RADS classification by one category. Diameter reduction after treatment should not be considered to be an AF favoring benignity.

For tumors that decrease in size following resorption of an acute bleed, diameter reduction should not be used as an AF favoring benignity. Some tumors may shrink slightly, possibly due to the resorption of hemorrhage or the development of fibrosis. Thus, diameter reduction does not guarantee that an observation is benign.

\section{Differences in AFs between Imaging Modalities}

Not every AF can be readily evaluated with each imaging modality. AFs can be grouped into four categories: (1) features that can be evaluated approximately equally well with all modalities; (2) features that can be better evaluated with CT and MRI than with US/CEUS; (3) features that can be evaluated with only one modality, generally MRI; and (4) features that can be evaluated with US only.

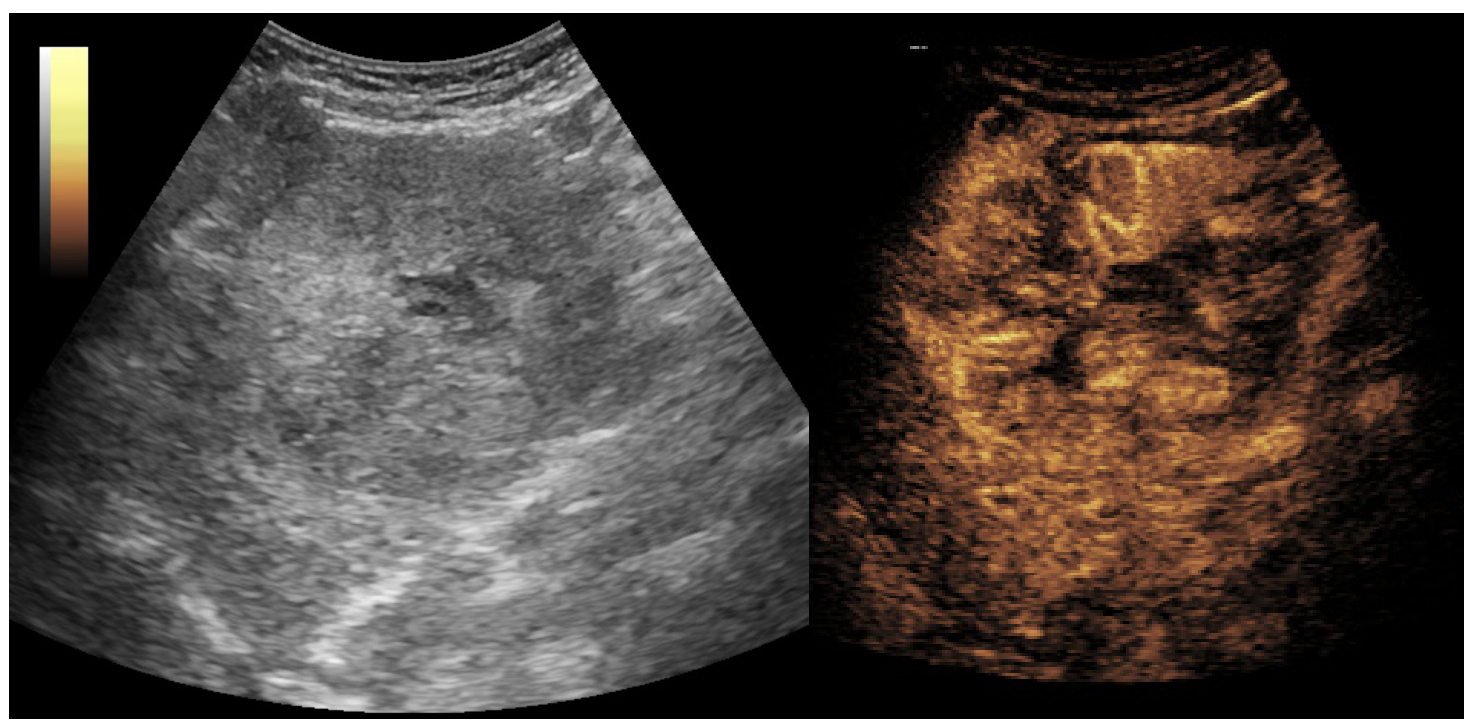

Fig. 1. Mosaic appearance. The patient is a 63-year-old man with hepatitis C virus/alcoholic cirrhosis and hepatocellular carcinoma. An arterial-phase image shows heterogeneous enhancement of this 8.5-cm LR-5 lesion. 
Features That Can be Evaluated Equally with All Modalities Mosaic appearance is defined as the presence of randomlydistributed nodules and compartments inside an observation, where those nodules and compartments display different levels of enhancement, intensity, attenuation, and/or echogenicity. As stated previously, mosaic appearance is an AF that favors HCC (Fig. 1), and nodule-in-nodule appearance (e.g., a hyperenhancing nodule developing inside an isoenhancing nodule) is a subtype of mosaic appearance (Fig. 2).

\section{Features That Can Be Better Evaluated with CT or MRI Than with US/CEUS}

Features for which CT or MRI evaluation is preferable over US/ CEUS imaging are subthreshold growth, corona enhancement,

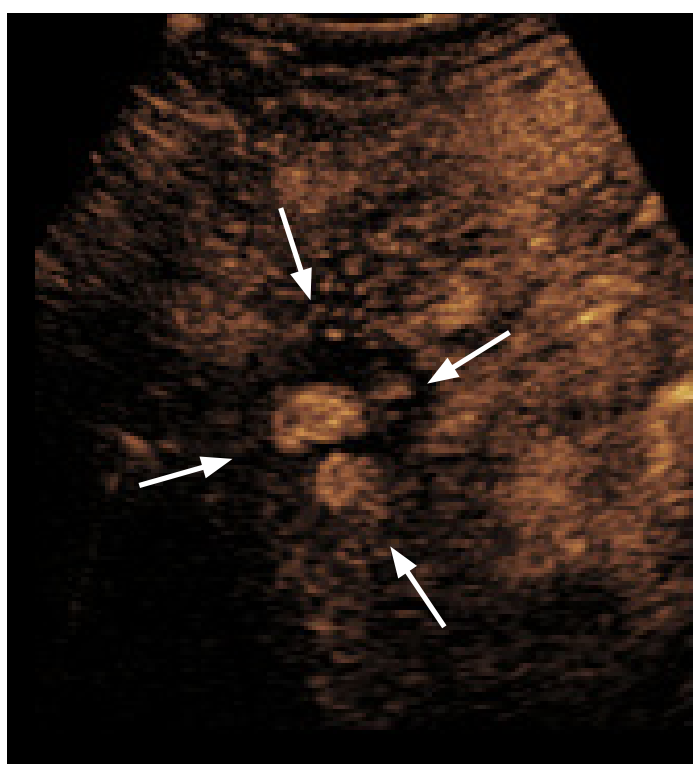

A

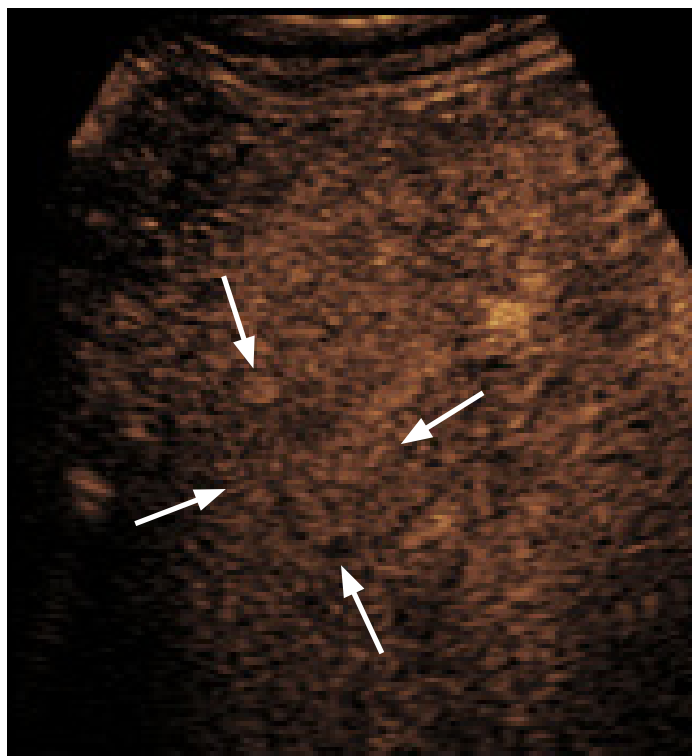

C

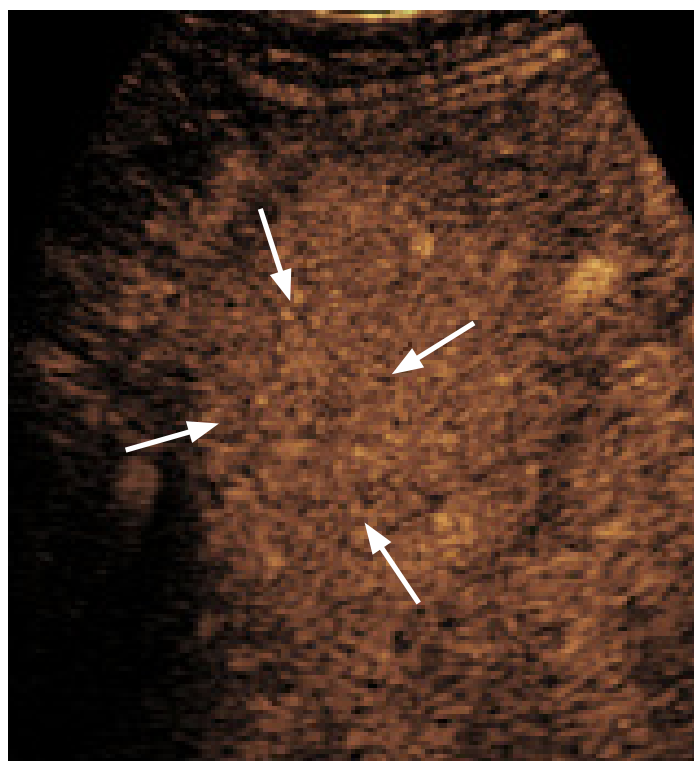

B

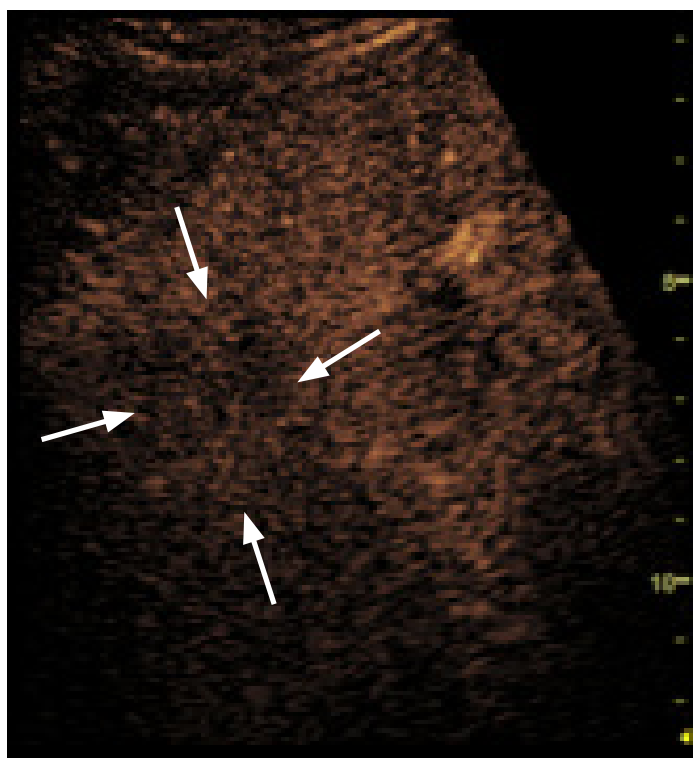

D

Fig. 2. Nodule-in-nodule appearance.

The patient is a 69-year-old man with alcoholic cirrhosis and an LR-4 lesion on magnetic resonance imaging. A. Contrast-enhanced ultrasound shows a 32-mm nodule with a nodule-in-nodule appearance in the arterial phase (arrows). B-D. The entire nodule (arrows) displays isoenhancement in the portal venous phase at 1 minute (B) and 2 minutes (C) and shows late and mild washout at 3 minutes (D). With regard to appearance, this is typical of a well-differentiated hepatocellular carcinoma focus developed in a dysplastic nodule. 
the presence of a non-enhancing capsule, and enhancement that parallels that of the blood pool.

\section{Features That Can Be Evaluated with Only One Modality, Generally MRI}

Some AFs can only be evaluated with MRI or can be better assessed with MRI than with other imaging modalities. This is due to the higher soft tissue resolution of MRI and to the technique's ability to employ multiple contrast media (e.g., hepatobiliary-specific contrast media, such as gadoxetic acid).

These AFs are as follows: T2-weighted imaging features, such as marked T2 hyperintensity (an AF favoring benignity) and mildmoderate $\mathrm{T} 2$ hyperintensity (an AF favoring malignancy in general); restricted diffusion; hypointensity of the nodule in the transitional and hepatobiliary phase after injection of liver-specific contrast media; the presence of fat or fatty sparing inside the nodule; the presence of iron or iron sparing inside a focal mass; and the presence of blood products inside or outside an untreated mass.

\section{Features That Can Be Evaluated with US Only}

US can also provide information which is not obtainable by other imaging techniques [26-28], such as US visibility as a discrete nodule, which is an AF that favors malignancy in general. This AF was not listed in previous versions of the LI-RADS, but was included in the 2017 version of the LI-RADS criteria. Darnell et al. [29] reported that $96 \%$ of LR- 4 and $69 \%$ of LR-3 observations that were visible on US were determined to be HCC nodules.

Interpreters may, at their discretion and after review of CEUS images, apply AFs to adjust the LI-RADS category as follows. The presence of one or more AFs that favor malignancy may be used to upgrade by one category to a maximum classification of LR-4; however, AFs cannot be used to upgrade the category to LR-5. This rule is designed to preserve high specificity for the definite diagnosis of $\mathrm{HCC}$, to prevent misdiagnosis (i.e., the false-positive diagnosis of HCC in patients under consideration for liver transplantation), and to maintain congruency with Organ Procurement and Transplantation Network criteria, which do not recognize AFs. The presence of one or more AFs that favor benignity may be used to downgrade the LIRADS classification by one category. If conflicting AFs are present (i.e., one or more favoring malignancy and one or more favoring benignity), the category should not be adjusted.

\section{Other Potential CEUS Features}

At the present time, the imaging features below are not recognized as LI-RADS AFs.

\section{Central or Eccentric Arterial Blood Supply}

A nodule may fill with contrast medium from the center to the periphery, originating from a large central artery [30,31]. This blood supply is characteristic of focal nodular hyperplasia (FNH) and can be considered an AF favoring benignity. The presence of this feature is also important to differentiate hepatocellular adenoma from FNH [31-33]. The individual vessels can be best visualized using CEUS with strict intravascular contrast agents [34]. FNH in cases of liver cirrhosis is rarely reported; therefore, the feature is of little value in daily practice except for patients who are at risk for HCC due to hepatitis B without cirrhosis [35].

\section{Conclusion}

In preparing this manuscript, a few questions were discussed but not yet answered. One of these questions was whether supportive AFs exist for conventional non-enhanced imaging techniques, including CT, MRI, conventional B-mode (gray-scale) US, and Doppler techniques. Until now, most evidence has been published on AFs in the setting of MRI, and little information has been published on the use of different equipment and differences in contrast agents used.

Future studies should focus on the similarities or differences between AFs on CEUS, CT, and MRI and their clinical utility. In addition, AFs should be examined separately with SonoVue/Lumason and Sonazoid contrast agents and compared.

ORCID: Christoph F. Dietrich: https://orcid.org/0000-0001-6015-6347; Yi Dong; https://orcid.org/0000-0002-0212-1477; Yuko Kono: https://orcid.org/0000-00029357-4104; Cosmin Caraiani: https://orcid.org/0000-0003-4453-2896; Claude B. Sirlin: https://orcid.org/0000-0002-6639-9072; Xin-Wu Cui: https://orcid.org/00000003-3890-6660; An Tang: https://orcid.org/0000-0001-8967-5503

\section{Author Contributions}

Conceptualization: Dietrich CF, Dong Y, Kono Y, Caraiani C, Sirlin CB, Cui XW, Tang A. Data acquisition: Dietrich CF, Dong Y, Kono Y, Caraiani C, Sirlin CB, Cui XW, Tang A. Data analysis or interpretation: Dietrich CF, Dong Y, Kono Y, Caraiani C, Sirlin CB, Cui XW, Tang A. Drafting of the manuscript: Dietrich $C F$, Dong $Y$, Kono $Y$, Caraiani $C$, Sirlin CB, Cui XW, Tang A. Critical revision of the manuscript: Dietrich CF, Dong Y, Kono Y, Caraiani C, Sirlin CB, Cui XW, Tang A. Approval of the final version of the manuscript: all authors.

\section{Conflict of Interest}

No potential conflict of interest relevant to this article was reported. 


\section{References}

1. Villanueva A. Hepatocellular carcinoma. N Engl J Med 2019;380:1450-1462.

2. Dietrich CF, Kono Y, Cosgrove D, Jang HJ, Kim TK, Piscaglia F, et al. Solutions in contrast imaging: contrast-enhanced ultrasound: Liver Imaging Reporting and Data System (CEUS LI-RADS) [Internet]. Milano: Sintesi InfoMedica, 2016 [cited 2019 Oct 1]. Available from: http:// www.solutionsincontrastimaging.com/upload/pdf_riviste/32016.pdf.

3. Piscaglia F, Wilson SR, Lyshchik $A$, Cosgrove D, Dietrich CF, Jang HJ, et al. American College of Radiology contrast enhanced ultrasound Liver Imaging Reporting and Data System (CEUS LI-RADS) for the diagnosis of hepatocellular carcinoma: a pictorial essay. Ultraschall Med 2017;38:320-324.

4. Wilson SR, Lyshchik A, Piscaglia F, Cosgrove D, Jang HJ, Sirlin C, et al. CEUS LI-RADS: algorithm, implementation, and key differences from CT/MRI. Abdom Radiol (NY) 2018;43:127-142.

5. Kono Y, Lyshchik A, Cosgrove D, Dietrich CF, Jang HJ, Kim TK, et al. Contrast Enhanced Ultrasound (CEUS) Liver Imaging Reporting and Data System $\left(\right.$ LI-RADS $\left.^{\circledR}\right)$ : the official version by the American College of Radiology (ACR). Ultraschall Med 2017;38:85-86.

6. Kim TK, Noh SY, Wilson SR, Kono Y, Piscaglia F, Jang HJ, et al. Contrast-enhanced ultrasound (CEUS) Liver Imaging Reporting and Data System (LI-RADS) 2017: a review of important differences compared to the CT/MRI system. Clin Mol Hepatol 2017;23:280289.

7. Lyshchik A, Kono Y, Dietrich CF, Jang HJ, Kim TK, Piscaglia F, et al. Contrast-enhanced ultrasound of the liver: technical and lexicon recommendations from the ACR CEUS LI-RADS working group. Abdom Radiol (NY) 2018;43:861-879.

8. Tang A, Bashir MR, Corwin MT, Cruite I, Dietrich CF, Do RK, et al. Evidence supporting LI-RADS major features for CT- and MR imaging-based diagnosis of hepatocellular carcinoma: a systematic review. Radiology 2018;286:29-48.

9. Wang JY, Feng SY, Yi AJ, Zhu D, Xu JW, Li J, et al. Comparison of contrast-enhanced ultrasound versus contrast-enhanced magnetic resonance imaging for the diagnosis of focal liver lesions using the Liver Imaging Reporting and Data System. Ultrasound Med Biol 2020;46:1216-1223.

10. Dong Y, Wang WP, Mao F, Zhang Q, Yang D, Tannapfel A, et al. Imaging features of fibrolamellar hepatocellular carcinoma with contrast-enhanced ultrasound. Ultraschall Med 2020 Feb 26 [Epub]. https://doi.org/10.1055/a-1110-7124.

11. Wang JY, Feng SY, Xu JW, Li J, Chu L, Cui XW, et al. Usefulness of the contrast-enhanced ultrasound Liver Imaging Reporting and Data System in diagnosing focal liver lesions by inexperienced radiologists. J Ultrasound Med 2020 Feb 20 [Epub]. https://doi.org/10.1002/jum.15242.

12. Kono Y, Sirlin CB, Fetzer DT, Kim TK, Rodgers SK, Piscaglia F, et al. Time to clarify common misconceptions about the Liver Imaging
Reporting and Data System for contrast-enhanced US. Radiology 2020;295:245-247.

13. Chernyak V, Tang A, Flusberg M, Papadatos D, Bijan B, Kono Y, et al. LI-RADS ${ }^{\circledast}$ ancillary features on CT and MRI. Abdom Radiol (NY) 2018;43:82-100.

14. Elsayes KM, Hooker JC, Agrons MM, Kielar AZ, Tang A, Fowler KJ, et al. 2017 Version of LI-RADS for CT and MR imaging: an update. Radiographics 2017;37:1994-2017.

15. Rodgers SK, Fetzer DT, Gabriel H, Seow JH, Choi HH, Maturen KE, et al. Role of US LI-RADS in the LI-RADS algorithm. Radiographics 2019:39:690-708.

16. Dietrich CF, Mertens JC, Braden B, Schuessler G, Ott M, Ignee A. Contrast-enhanced ultrasound of histologically proven liver hemangiomas. Hepatology 2007;45:1139-1145.

17. Hirche TO, Ignee A, Hirche H, Schneider A, Dietrich CF. Evaluation of hepatic steatosis by ultrasound in patients with chronic hepatitis $\mathrm{C}$ virus infection. Liver Int 2007;27:748-757.

18. Dietrich CF, Wehrmann T, Zeuzem S, Braden B, Caspary WF, Lembcke B. Analysis of hepatic echo patterns in chronic hepatitis $C$. Ultraschall Med 1999:20:9-14.

19. Cruite I, Santillan C, Mamidipalli A, Shah A, Tang A, Sirlin CB. Liver Imaging Reporting and Data System: review of ancillary imaging features. Semin Roentgenol 2016;51:301-307.

20. Chernyak $V$, Kobi M, Flusberg M, Fruitman KC, Sirlin CB. Effect of threshold growth as a major feature on LI-RADS categorization. Abdom Radiol (NY) 2017:42:2089-2100.

21. Granata V, Fusco R, Avallone $A$, Catalano $O$, Filice $F$, Leongito $M$, et al. Major and ancillary magnetic resonance features of LI-RADS to assess HCC: an overview and update. Infect Agent Cancer 2017;12:23.

22. Stevens WR, Gulino SP, Batts KP, Stephens DH, Johnson CD. Mosaic pattern of hepatocellular carcinoma: histologic basis for a characteristic CT appearance. J Comput Assist Tomogr 1996;20:337-342.

23. Choi BI, Takayasu K, Han MC. Small hepatocellular carcinomas and associated nodular lesions of the liver: pathology, pathogenesis, and imaging findings. AJR Am J Roentgenol 1993;160:1177-1187.

24. Granata V, Fusco R, Avallone A, Filice F, Tatangelo F, Piccirillo $M$, et al. Critical analysis of the major and ancillary imaging features of LI-RADS on 127 proven HCCs evaluated with functional and morphological MRI: Lights and shadows. Oncotarget 2017:8:51224-51237.

25. Narsinh KH, Cui J, Papadatos D, Sirlin CB, Santillan CS. Hepatocarcinogenesis and LI-RADS. Abdom Radiol (NY) 2018;43:158-168.

26. Dietrich CF, Averkiou M, Nielsen MB, Barr RG, Burns PN, Calliada $F$, et al. How to perform contrast-enhanced ultrasound (CEUS). Ultrasound Int Open 2018;4:E2-E15.

27. Dietrich CF, Averkiou MA, Correas JM, Lassau N, Leen E, Piscaglia 
F. An EFSUMB introduction into dynamic contrast-enhanced ultrasound (DCE-US) for quantification of tumour perfusion. Ultraschall Med 2012;33:344-351.

28. Ignee A, Atkinson NS, Schuessler G, Dietrich CF. Ultrasound contrast agents. Endosc Ultrasound 2016;5:355-362.

29. Darnell A, Forner A, Rimola J, Reig M, Garcia-Criado A, Ayuso C, et al. Liver Imaging Reporting and Data System with MR imaging: evaluation in nodules $20 \mathrm{~mm}$ or smaller detected in cirrhosis at screening US. Radiology 2015;275:698-707.

30. Dietrich CF, Schuessler G, Trojan J, Fellbaum C, Ignee A. Differentiation of focal nodular hyperplasia and hepatocellular adenoma by contrast-enhanced ultrasound. $\mathrm{Br} J$ Radiol 2005;78:704-707.

31. Dietrich CF, Tannapfel A, Jang HJ, Kim TK, Burns PN, Dong Y.
Ultrasound imaging of hepatocellular adenoma using the new histology classification. Ultrasound Med Biol 2019;45:1-10.

32. Schreiber-Dietrich DG, Leuschner I, Tannapfel A, Franke D, Stenzel M, Juengert J, et al. Primary liver tumours in childhood. Z Gastroenterol 2015;53:1267-1275.

33. Chiorean L, Cui XW, Tannapfel A, Franke D, Stenzel M, Kosiak W, et al. Benign liver tumors in pediatric patients: review with emphasis on imaging features. World J Gastroenterol 2015;21:8541-8561.

34. Kang HS, Kim BK, Shim CS. Focal nodular hyperplasia: with a focus on contrast enhanced ultrasound. Korean J Hepatol 2010;16:414417.

35. Ignee A, Weiper D, Schuessler G, Teuber G, Faust D, Dietrich CF. Sonographic characterisation of hepatocellular carcinoma at time of diagnosis. Z Gastroenterol 2005;43:289-294. 